\title{
Análisis del comportamiento financiero de las universidades intervenidas por el consejo de educación superior (2013 - 2016)
}

\section{Analysis of the financial behavior of the universities intervened by the Higher Education Council (2013 - 2016)}

Doris Cevallos Zambrano

Universidad Laica Eloy Alfaro de Manabí, Ecuador

Mayra Liuviana Vega Chica

Universidad de Especialidades Espíritu Santo, Ecuador

Autor por correspondencia: mvegach@uees.edu.ec; superdoris99@hotmail.com

Fecha de recepción: 06 de julio de 2018 - Fecha de aceptación: 01 de diciembre de 2018

\section{Resumen}

El trabajo pertenece al campo de la Educación Superior en Ecuador. Centrándose en el comportamiento financiero de las universidades inmersas en un proceso de intervención por parte del Consejo de Educación Superior. Pretende analizar el comportamiento financiero de las universidades intervenidas por el Consejo de Educación Superior del Ecuador. La información es primaria recabada en cada una de las instituciones en estudio, mediante la investigación de campo y directa, cuyos resultados evidencian la inversión por parte del estado y la subejecución de los presupuestos universitarios con índices que requieren mayor atención de las autoridades universitarias, dichos resultados, pretenden aportar académicamente al fortalecimiento institucional de las universidades públicas del país.

Palabras claves: comportamiento financiero; administración pública; universidades; intervención; análisis

\begin{abstract}
The work belongs to the field of Higher Education in Ecuador. Focusing on the financial behavior of universities immersed in a process of intervention by the Council of Higher Education. It intends to analyze the financial behavior of the universities intervened by the Council of Higher Education of Ecuador. The information is primary collected in each of the institutions under study, through field and direct research, whose results show the investment by the state and the under-execution of university budgets with rates that require greater attention from university authorities, such results, are intended to contribute academically to the institutional strengthening of public universities in the country.
\end{abstract}

Key words: financial behavior; public administration; universities; intervention; analysis 


\section{Introducción}

El artículo 197 de la LOES define al proceso de intervención, e indica que con ello el Estado garantiza el derecho irrenunciable de las personas a una educación de calidad de acuerdo con lo establecido en la Constitución de la República del Ecuador. La intervención no suspende el funcionamiento de la universidad o escuela politécnica intervenida, ni a sus autoridades, busca elevar la capacidad de gestión institucional a través de la normalización, evitando perjuicios a la comunidad universitaria o politécnica. (Asamblea Nacional, 2010b).

Analizar el comportamiento financiero de la universidades intervenidas en Ecuador, resulta relevante académica y científicamente dado que no existe investigaciones al respecto; debido a que la LOES es relativamente nueva, aprobada en 2010 y realizando su primera actuación de intervención en septiembre de 2013 en la UNESUM, posteriormente se han intervenido cuatro universidades más, por ello se investigó a las cinco universidades. Sin embargo, a la fecha del presente trabajo el proceso ha concluido en cuatro y solamente se mantiene en una como es la Universidad Nacional de Loja.

El proceso de intervención como lo plantea la LOES en Ecuador, no existe como figura de gestión o gobernanza en otros países, quizá algunos modelos que buscan mejorar universidades en conflicto. Por ende, se convierte la figura de intervención, en una figura que geográficamente es localizada en Ecuador y que aportará elementos pocos conocidos en el marco de la educación superior.

Desde el punto de vista social, las universidades cumplen un rol fundamental en la construcción de mejores sociedades, por ello las personas tienen puestas sus esperanzas en las instituciones de educación superior. Las universidades en conflicto, afectan social y políticamente en su caso, porque todas las universidades intervenidas hasta la fecha, son públicas y de peso político para el gobierno; pues la gestión de ellas, será favorable o desfavorable para la imagen del gobierno en ese territorio.

El análisis del comportamiento financiero en las universidades intervenidas se hace considerando, tanto la información primaria de fuentes oficiales, como la aplicación de un trabajo de campo representativo que involucró a los principales actores, tanto del equipo rector de las universidades, como de los equipos de las comisiones de intervención de las universidades.

El presente documento pretende analizar el comportamiento financiero de las universidades intervenidas por el Consejo de Educación Superior del Ecuador, durante el periodo 2013 al 2016. Mediante información primaria recabada en cada una de las instituciones en estudio, la investigación ha sido de campo y directa, misma que posteriormente ha sido sistematizada y analizada.

El documento aplica método de análisis deductivo partiendo de un apartado respecto a la educación superior en Ecuador, continúa con el análisis de las universidades intervenidas, contextualizando las finanzas públicas y su manejo teórico en el sector público de las 
universidades en Ecuador, en la parte final se explica la metodología aplicada y los resultados obtenidos que son los presupuestos de las universidades estudiadas.

\section{Contexto de la educación superior en Ecuador}

La Constitución Política de la República del Ecuador (2008), en el art. 26 indica que la educación es un derecho de las personas a lo largo de su vida y un deber ineludible e inexcusable del Estado. Constituye un área prioritaria de la política pública y de la inversión estatal, garantiza la igualdad e inclusión social y condición indispensable para el buen vivir. Las personas, las familias y la sociedad tienen el derecho y la responsabilidad de participar en el proceso educativo.

El artículo 355 ibídem determina que el Estado reconocerá a las universidades y escuelas politécnicas autonomía académica, administrativa, financiera y orgánica, acorde con los objetivos del régimen de desarrollo y los principios establecidos en la Constitución.

El desarrollo de la educación en Ecuador en todos sus niveles ha venido favoreciendo a una gran cantidad de niños, jóvenes y adultos lo cual ha significado múltiples beneficios sociales que han repercutido positivamente en el crecimiento y desarrollo del país.

La reforma a la educación superior ha generado logros importantes, sobre todo en términos de la inversión y distribución de recursos, la democratización del acceso, el desarrollo de normativa, la mejora de la calidad de la enseñanza, entre otros, lo que se evidencia con datos de la UNESCO (2010) en los que se observa que Ecuador es el país de América del Sur que más recursos destina a Educación Superior en relación a su economía.

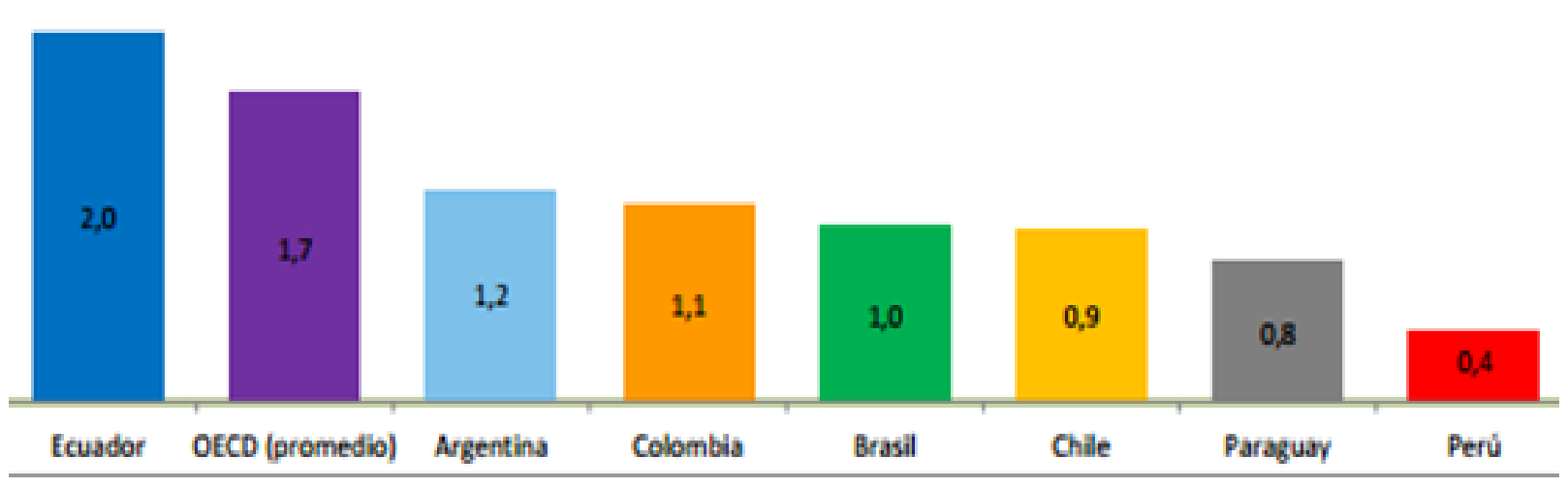

Gráfico 1: Gasto público en Educación Superior (Porcentaje PIB)

Fuente: (UNESCO, 2010a) Total Public Expenditure con Higher Education as \% of GDP.

La universidad ecuatoriana debe prepararse para enfrentar los enormes desafíos que le plantea un entorno altamente cambiante derivado de la globalización y exigencias de la sociedad del conocimiento. Para ello debe encarar exitosamente las dificultades derivadas de la búsqueda de una mejor calidad de educación basada en la pertinencia de los planes curriculares, mejora de los servicios e investigación que responda a las demandas sociales. El Estado ecuatoriano no ha estado ajeno al rol que las actuales circunstancias lo demandan y en este contexto se expidió la 
Ley Orgánica de Educación Superior (LOES) que norma y regula el desempeño de las Instituciones de Educación Superior (IES).

La LOES, reafirma la gratuidad de la educación superior pública; reitera la necesidad de articular la educación con las metas de desarrollo local y nacional; plantea la necesidad de asegurar la calidad de la educación superior; busca la democratización del acceso a la universidad, pero también de las prácticas gubernativas y administrativas de las instituciones; consolida la educación superior como un bien público y plantea la necesidad de una mayor regulación estatal para asegurar que esto se cumpla. Para lograr su cometido, la LOES creó tres instituciones destinadas a planificar y ejecutar las políticas públicas, y regular el Sistema de Educación Superior:

La Secretaría Nacional de Educación Superior, Ciencia, Tecnología e Innovación (SENESCYT):

Ministerio rector de la política pública de educación superior.

El Consejo de Educación Superior (CES): autoriza la apertura de carreras y programas, expide normativas y reglamentos para regular el campo, y sanciona las ilegalidades; y,

El Consejo de Evaluación, Acreditación, y Aseguramiento de la Calidad de la Educación Superior (CEAACES): asume la evaluación, acreditación y categorización institucional y de carreras.

\section{Las universidades intervenidas en Ecuador}

El artículo 199 de la LOES, prescribe cuáles son las causales de intervención:

a) La violación o el incumplimiento de las disposiciones de la Constitución de la República, de la Ley, su Reglamento General, los reglamentos, resoluciones y demás normatividad que expida el Consejo de Educación Superior, y el estatuto de cada institución.

b) La existencia de irregularidades académicas, administrativas o económico financieras, establecidas en la normatividad vigente que atenten contra el normal funcionamiento institucional.

c) La existencia de situaciones de violencia que atenten contra el normal funcionamiento institucional y los derechos de la comunidad universitaria o politécnica, que no puedan ser resueltas bajo los mecanismos y procedimientos establecidos por las instituciones de educación superior. (Asamblea Nacional, 2010b)

El artículo 197 de la Ley ibídem, define al proceso de intervención e indica que con ello el Estado garantiza el derecho irrenunciable de las personas a una educación de calidad de acuerdo con lo establecido en la Constitución de la República del Ecuador. La intervención no suspende el funcionamiento de la universidad o escuela politécnica intervenida, ni a sus autoridades, busca elevar la capacidad de gestión institucional a través de la normalización, evitando los perjuicios a la comunidad universitaria o politécnica. 
Durante el proceso de intervención por parte CES, la universidad intervenida continúa funcionando con normalidad con su equipo rector, con la diferencia que el CES como órgano rector de la educación superior nombra a una Comisión de Intervención y Fortalecimiento Institucional (CIFI) para velar por el correcto funcionamiento de la universidad.

La miembros de la comisión de Intervención y Fortalecimiento institucional que nombre el CES, se conforma por un Presidente que debe cumplir los mismos requisitos que un rector, y un miembro especialista por cada área intervenida, un especialista académico, un especialista administrativo financiero y un jurídico, con opción a tener un equipo asesor. En la práctica los directivos de la universidad deciden el rumbo de la misma, sin embargo toda decisión que comprometa el patrimonio institucional y resoluciones del Órgano Colegiado Académico Superior (OCAS) deben ser conocidas y aprobadas por la Comisión previo a su ejecución, caso contrario las decisiones tomadas por la universidad carecen de legalidad.

Para regular éste proceso el CES expide el Reglamento de Creación, Intervención y Suspensión de Universidades y Escuelas Politécnicas, el cual delimita desde las causales de Intervención, el funcionamiento de las comisiones, atribuciones, y demás, que permitan velar por el buen funcionamiento de la institución intervenida.

Inicialmente, dicho reglamento consideraba un periodo de intervención de un año con opción de prórroga de un año adicional, posteriormente en base a las realidades encontradas en las universidades el CES modifica el reglamento, amparando al periodo de intervención a dos años con opción prorrogable de dos años más, significándose que una intervención puede extenderse hasta cuatro años, de acuerdo a la complejidad de su problemática.

Para el caso de estudio se tomaron en consideración cinco universidades intervenidas hasta el año 2016, a continuación se hace una breve explicación de cada una de ellas: 


\section{Tabla 1: Caracterización de las universidades intervenidas en Ecuador}

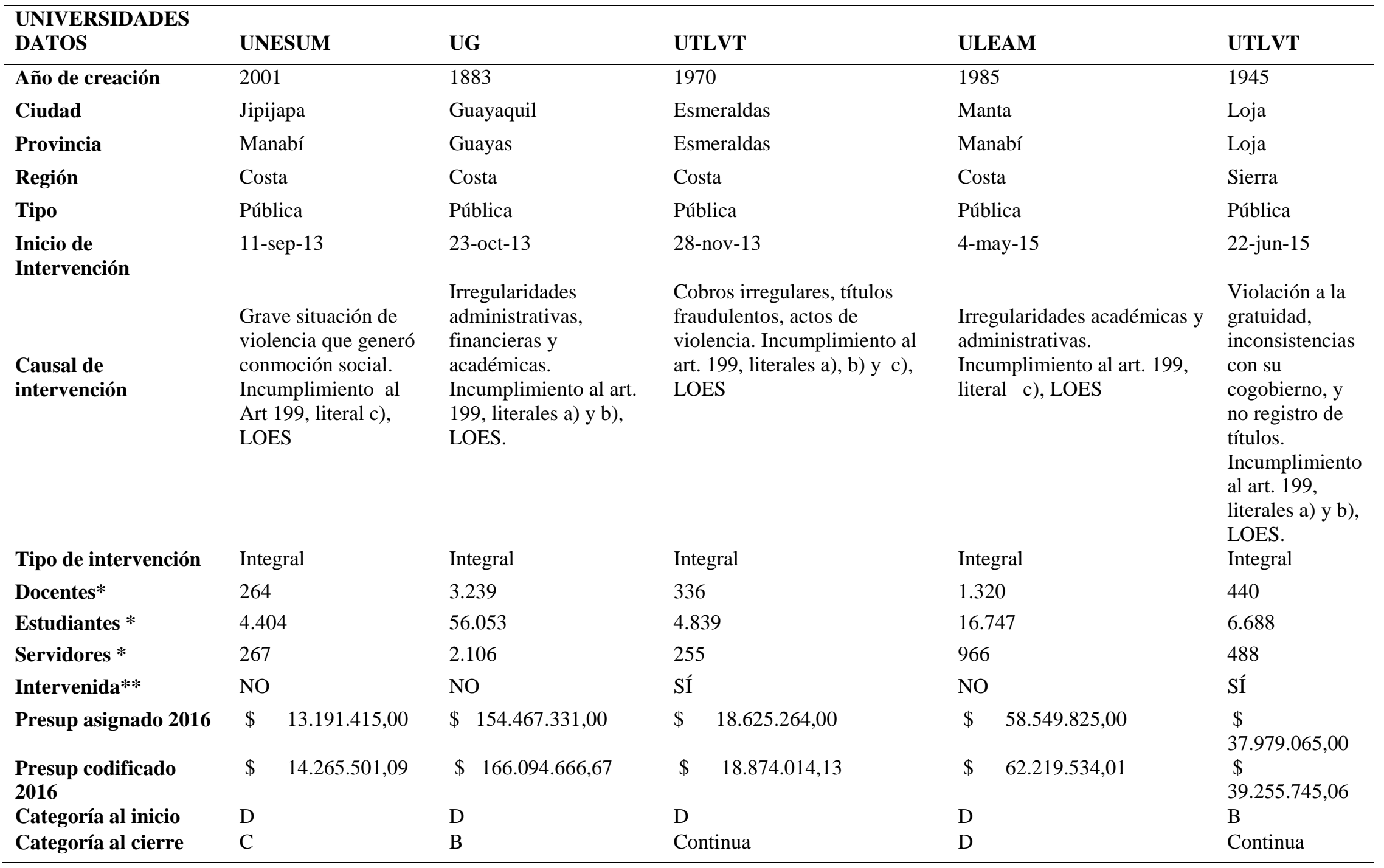

Fuente: Elaboración propia

*Cantidades periodo 2016 (2) - **A enero 2017 
Universidad Estatal del Sur de Manabí: Se ubica en la provincia de Manabí, creada en el 2001, intervenida el 11 de septiembre de 2013 mediante Resolución RPC-SE-09-N․035-2013, es una universidad en categoría D la última categoría de acuerdo al CEAACES, tiene 4.500 estudiantes, 270 profesores y maneja un presupuesto de 11965260.00 USD en el año 2015. La causal de intervención fue por la grave situación de violencia que generó conmoción social y es de conocimiento público, y que derivó la detención de quien dirigía en calidad de rector a la universidad y sus dos hijos.

Universidad de Guayaquil: Se ubica en la provincia del Guayas, intervenida el 23 de octubre de 2013 mediante Resolución RPC-SO-41-N.419-2013, es considerada la universidad más grande del país ubicada en categoría D, la más antigua de Guayaquil creada en 1883, con alrededor de 70000 estudiantes, 2800 profesores y con un presupuesto de 183968106.00 USD en el año 2015, lo que la convierte en la universidad que más recursos del estado recibe. La causal de intervención fue por las irregularidades evidenciadas en el área administrativa, financiera y académica, por tanto es una intervención integral.

Universidad Luis Vargas Torres: Se ubica en la provincia de Esmeraldas, creada en 1970, intervenida el 28 de noviembre de 2013 mediante Resolución RPC-SE-14-Nº.053-2013, es también universidad categoría D, tiene 10500 estudiantes y con un presupuesto para el año 2015 de 20824762.00 USD, la causal de intervención fue integral dado que se identificó cobros irregulares en las extensiones, emisión de títulos fraudulentos, irregularidades en la elección de representantes estudiantiles, así como actos de violencia.

Universidad Laica Eloy Alfaro de Manabí: Ubicada en la provincia de Manabí, creada en 1985, universidad de categoría D, se convierte en la segunda intervenida en la provincia el 4 de mayo de 2015 mediante Resolución RPC-SE-02-N $.003-2015$, por irregularidades académicas y administrativas, cuenta con 21000 estudiantes, 1200 profesores y un presupuesto para el 2015 de 59726765.00 USD.

Universidad Nacional de Loja: intervenida el 22 de junio de 2015 mediante Resolución RPC-SE-04-Nº09-2015, creada en 1945, ubicada en categoría B de acuerdo al CEAACES, su causal de intervención violación a la gratuidad, al cogobierno, cierre de oferta vigente, y no registro de títulos, cuenta con 19000 estudiantes y 850 profesores, y un presupuesto de 40559 876.00 USD en el año 2015, ha sido la última universidad intervenida en Ecuador.

\section{Finanzas públicas}

Las finanzas se definen como el arte y la ciencia de administrar el dinero, (Lawrence $\mathbf{J} \&$ Chad J, 2012, 3). Los estados financieros deben considerarse como un medio para comunicar información y no como un fin; su objetivo es proporcionar información sobre la situación financiera, los resultados de las operaciones, los flujos de efectivo y el movimiento en el capital contable de una entidad. (Leopoldo, 2012, 4).

A la palabra "finanzas" se le puede adicionar la palabra "públicas", con el propósito de distinguir las finanzas públicas estatales de las privadas. (Córdoba Padilla, 2014, 25) 
Se encuentra también la definición de hacienda pública, es así que se la considera como: "La disciplina de la Hacienda Pública describe y analiza los servicios, subvenciones y gastos sociales del Sector Público, así como los métodos para financiar estos gastos: los impuestos, el endeudamiento, la ayuda económica del extranjero y la creación de dinero...”. Y a ello agregaba: "La Hacienda Pública trata de un sistema de asignación de recursos que, aunque apenas utiliza el mecanismo de precios, usa el dinero en forma considerable”. Carl S. Shoup, citado por Asensio $(2015,29)$

"Las finanzas públicas estudian el progreso ingreso-gasto llevado a cabo por el Estado generalmente expresado en términos monetarios, en cuanto permite determinarla forma en que el Estado logra los fines perseguidos y las consecuencias - deseadas o no deseadas- de dicho proceso". (Nuñez, 1994, 30). En concordancia con Nuñez, (1994), aquellos fines que persiguen las finanzas públicas pretende establecer a nivel gubernamental el nivel de cumplimiento de los objetivos del gobierno.

"Las finanzas del Estado constituyen la actividad económica del sector público, con su particular y característica estructura que convive con la economía de mercado, de la cual obtiene los recursos y a la cual le presta un marco de acción". (Aquino, 2011) citado por (Córdoba Padilla, 2014)

Las finanzas del Estado, llamadas también "finanzas públicas", "hacienda pública” y "economía financiera", es una ciencia social, una ciencia de la cultura, situada entre tres grandes campos científicos: la economía, el derecho y la política. Es una ciencia que estudia una de las más importantes funciones o actividades del estado: la "actividad financiera", que es la gestión que realiza el ente público para lograr los medios económicos que le permitan lograr sus fines. (Córdoba Padilla, 2014).

Dicho por otro autor las finanzas públicas es la forma en que el Estado asigna los recursos (gasto), y la manera como los capta (ingreso), constituye el objetivo de la teoría de las finanzas públicas. El presupuesto es el instrumento de manejo financiero más importante de la política fiscal, donde se programa y registra el gasto público y su forma de financiación y donde se establecen reglas para su ejecución. (Córdoba Padilla, 2014)

\section{El Balance general}

También llamado estado de situación financiera, este estado financiero presenta información que permite hacer un análisis de la posición financiera, el cual consiste en diagnosticar la estructura financiera y la liquidez de la empresa. (Leopoldo, 2012, 5)

\section{La información financiera y la toma de decisiones}

Tanto para administrar una empresa como para conocerla es necesario tener información financiera, dado que es primordial para la administración y el conocimiento de un negocio; pero el uso de dicha información se hace desde diferentes puntos de vista dependiendo de los usuarios, ya que cada uno puede tener diferentes objetivos, problemas por resolver e incluso oportunidades por lograr. (Leopoldo, 2012, 6) 


\section{Código orgánico de planificación y finanzas públicas}

En Ecuador existe el Código orgánico de planificación y finanzas públicas, que de acuerdo a su artículo 1, determina que su objeto es: El presente código tiene por objeto organizar, normar y vincular el Sistema Nacional Descentralizado de Planificación Participativa con el Sistema Nacional de Finanzas Públicas, y regular su funcionamiento en los diferentes niveles del sector público, en el marco del régimen de desarrollo, del régimen del buen vivir, de las garantías y los derechos constitucionales. (Asamblea Nacional, 2010a)

\section{Ley Orgánica de Educación Superior}

Desde el artículo 20 y hasta el artículo 44 de la Ley Orgánica de Educación Superior vigente en el Ecuador desde el 12 de octubre de 2010, se describe claramente cómo se financiarán las instituciones de educación superior del sector público. (Asamblea Nacional, 2010b).

Art. 20.- Del Patrimonio y Financiamiento de las instituciones del sistema de educación superior.- En ejercicio de la autonomía responsable, el patrimonio y financiamiento de las instituciones del sistema de educación superior estará constituido por:

1. Los bienes muebles e inmuebles que al promulgarse esta Ley sean de su propiedad, y los bienes que se adquieran en el futuro a cualquier título, así como aquellos que fueron ofertados y comprometidos al momento de presentar su proyecto de creación;

2. Las rentas establecidas en la Ley del Fondo Permanente de Desarrollo Universitario y Politécnico (FOPEDEUPO);

3. Las asignaciones que han constado y las que consten en el Presupuesto General del Estado, con los incrementos que manda la Constitución de la República del Ecuador;

4. Las asignaciones que corresponden a la gratuidad para las instituciones públicas;

5. Los ingresos por matrículas, derechos y aranceles, con las excepciones establecidas en la Constitución y en esta Ley en las universidades y escuelas politécnicas públicas;

6. Los beneficios obtenidos por su participación en actividades productivas de bienes y servicios, siempre y cuando esa participación no persiga fines de lucro y que sea en beneficio de la institución;

7. Los recursos provenientes de herencias, legados y donaciones a su favor;

8. Los fondos autogenerados por cursos, seminarios extracurriculares, programas de posgrado, consultorías, prestación de servicios y similares, en el marco de lo establecido en esta Ley; 
9. Los ingresos provenientes de la propiedad intelectual como fruto de sus investigaciones y otras actividades académicas;

10. Los saldos presupuestarios comprometidos para inversión en desarrollo de ciencia y tecnología y proyectos académicos y de investigación que se encuentren en ejecución no devengados a la finalización del ejercicio económico, obligatoriamente se incorporarán al presupuesto del ejercicio fiscal siguiente;

11. Los recursos obtenidos por contribuciones de la cooperación internacional; y,

12. Otros bienes y fondos económicos que les correspondan o que adquieran de acuerdo con la Ley.

Art. 21.- Acreditación de fondos.- Los fondos constantes en los literales 1), 2), 3), 4), 5), 6), 7), 8), 9), 10) y 11) del artículo anterior, que correspondan a las instituciones públicas, al igual que los recursos que correspondan a universidades particulares que reciben asignaciones y rentas del Estado, serán acreditados en las correspondientes subcuentas de la Cuenta Única del Tesoro Nacional.

Art. 23.- Garantía del financiamiento de las instituciones públicas de educación superior.De conformidad con la Constitución de la República del Ecuador y la presente Ley, el Estado garantiza el financiamiento de las instituciones públicas de educación superior, el que constará obligatoriamente en el Presupuesto General del Estado que se aprueba cada año.

Art. 24.- Distribución de los recursos.- Los recursos destinados anualmente por parte del Estado a favor de las universidades, escuelas politécnicas, institutos superiores técnicos, tecnológicos, pedagógicos, de artes y los conservatorios superiores públicos y particulares que reciban rentas y asignaciones del Estado, se distribuirán con base a criterios de calidad, eficiencia, equidad, justicia y excelencia académica, que entre otros parámetros prevalecerán los siguientes:

1. Número de estudiantes y costo por carrera y nivel;

2. Número, dedicación, título y experiencia docente en función de las evaluaciones pertinentes;

3. Clasificación académica y tipología de instituciones, carreras y programas;

4. Eficiencia en docencia e investigación y relación con el desarrollo nacional y regional;

5. Eficiencia terminal; $y$,

6. Eficiencia administrativa.

Los porcentajes correspondientes a cada parámetro de distribución se establecerán en el respectivo reglamento, y tendrán en cuenta: los lineamientos del Plan Nacional de Desarrollo, un 
sistema de incentivos orientados a la excelencia académica, el mejoramiento de la formación de las plantas de profesores e investigadores, el tipo de carrera, el fomento a la investigación, la innovación y el desarrollo tecnológico.

Se considerará como parámetro adicional, la vinculación de su oferta al desarrollo nacional o regional, a la creación de sinergias, asociaciones y/o fusiones con otras instituciones de educación superior de su región, y a la promoción de potencialidades territoriales.

Para la distribución de los recursos, la Secretaría Nacional de Educación Superior, Ciencia, Tecnología e Innovación, elaborará el informe respetivo que establezca la fórmula de distribución de los recursos, para aprobación del Consejo de Educación Superior. Una vez aprobada dicha fórmula, la Secretaría Nacional de Educación Superior, Ciencia, Tecnología e Innovación, distribuirá dichos recursos.

Las instituciones de educación superior que se crearen o que fueran incorporadas a la distribución de fondos por mandato de la Ley, recibirán la parte proporcional de los incrementos de las respectivas rentas, desde el año siguiente a su creación o incorporación.

La fórmula y las variables que considera la misma, se expresan a continuación:

$$
A_{i t F}=\left(\alpha \frac{\left(C_{i t}+\left(C_{i t-1}-C_{i t-2}\right)\right) N E_{i t-1}}{\sum_{i=1}^{n}\left(C_{i t}+\left(C_{i t-1}-C_{i t-2}\right)\right) N E_{i t-1}}+\beta E_{i t-1}+\gamma_{1} E F A D_{i t-1}+\gamma_{2} E F A_{i t-1}\right) A T_{t j}
$$

Donde:

AitF : Asignación total que recibe la institución de educación superior i, en el

Periodo $t$ a partir de la aplicación de la fórmula

i : Institución de educación superior.

t $\quad$ : año para el cual se distribuyen los recursos.

j : tipo de institución (pública de pregrado, postgrado, particulares, institutos)

a : Parámetro de distribución del criterio de "Calidad",

$\beta \quad$ : Parámetro de distribución del criterio de "Excelencia"

y1 : Parámetro de distribución del criterio de "Eficiencia Administrativa",

y2 : Parámetro de distribución del criterio de "Eficiencia Académica",

C : Calidad

NE : Número de estudiantes

E : Excelencia

EF AD : Eficiencia administrativa

EFA : Eficiencia académica, ajustado por Pertinencia

AT : Recursos a distribuirse según tipo de institución. 

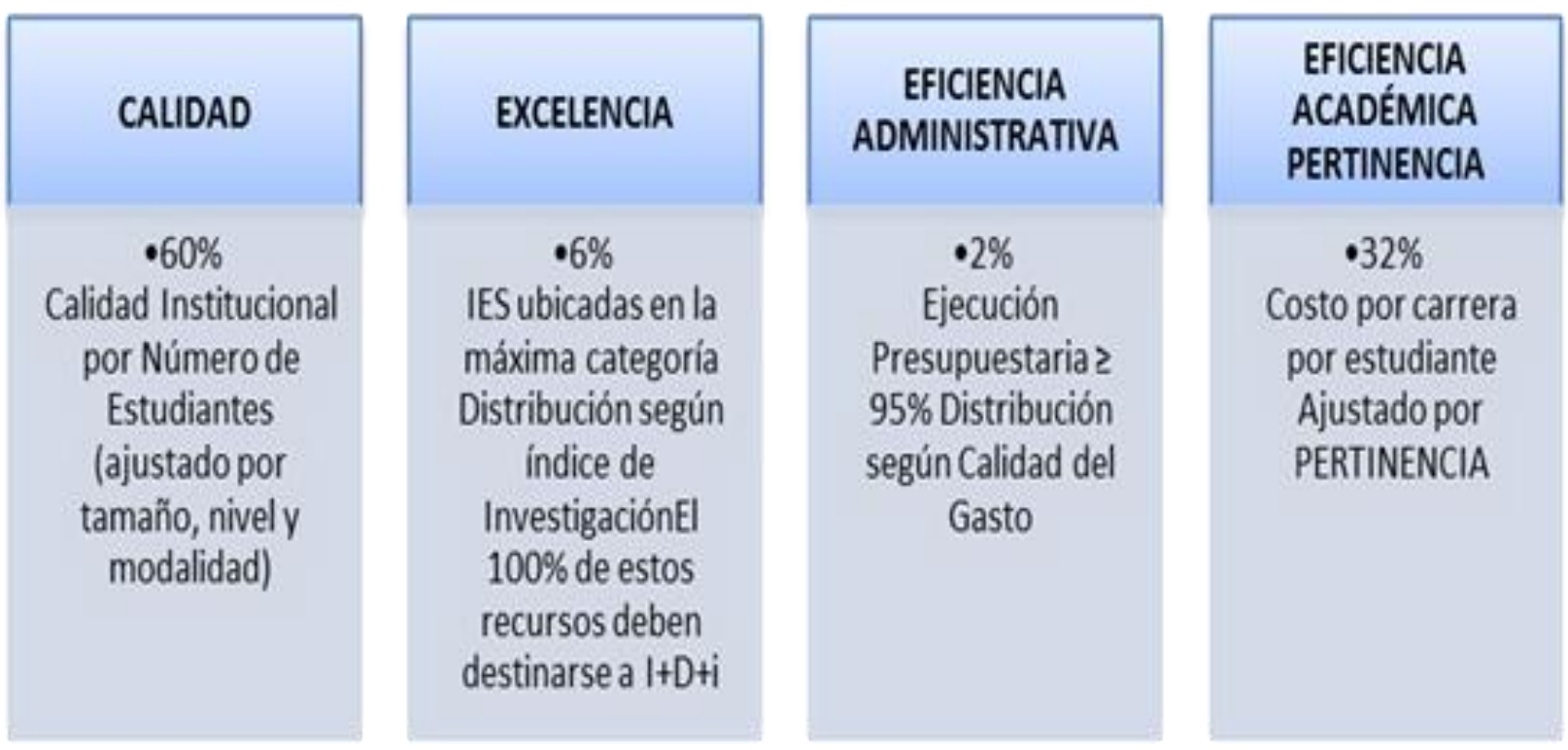

Gráfico 2: Parámetros de la fórmula de distribución de recursos Elaboración propia a partir de (Consejo de Educación Superior, 2017)

\section{Métodos}

Respecto al tipo de investigación es una investigación de carácter mixto, en primer momento por la connotación del tema en estudio se refleja la investigación cualitativa, comprendiéndola así porque "los investigadores cualitativos estudian la realidad en su contexto natural, tal y como sucede, intentando sacar sentido de, o interpretar los fenómenos de acuerdo con los significados que tienen para las personas implicadas". (Rodríguez G, Gil F, \& García J, 1996, 32). Teniendo un análisis documental del fenómeno en estudio, existe criterio para la investigación cualitativa y un enfoque con tendencia a la investigación cuantitativa se refiere al análisis de los datos financieros provistos por las universidades investigadas.

Para ir definiendo y comprendiendo el enfoque que tiene la investigación, se debe comprender cómo se originan las ideas de investigación mixtas "Las ideas constituyen el primer acercamiento a la realidad objetiva (desde la perspectiva cuantitativa), a la realidad subjetiva (desde la perspectiva cualitativa) o a la realidad intersubjetiva (desde la óptica mixta) que habrá de investigarse." (Hernández et al., 2010, 26).

De una manera más amplia y general se puede indicar que "el enfoque mixto de la investigación, que implica un proceso de recolección, análisis y vinculación de datos cuantitativos y cualitativos en un mismo estudio o una serie de investigaciones para responder a un planteamiento del problema”. (Hernández et al., 2010, 544).

\section{Formulación de objetivos}

\section{El objetivo general:}


Analizar el comportamiento financiero de las universidades intervenidas por el Consejo de Educación Superior del Ecuador.

Para ello se desglosan los objetivos específicos que pretenden lograr el cumplimiento del objetivo general.

- Caracterizar el sistema de educación superior en el Ecuador, poniendo énfasis en las universidades intervenidas.

- $\quad$ Comprender el funcionamiento de las universidades intervenidas.

- $\quad$ Comprender las finanzas públicas y su intervención en las universidades.

- $\quad$ Analizar las cedulas presupuestarias de cada universidad y evaluar sus hallazgos.

- Elaborar recomendaciones que aporten al fortalecimiento institucional

\section{Estrategia metodológica}

\section{Análisis documental}

El análisis documental una estrategia utilizada durante toda la investigación, sirve para complementar los hallazgos, de ésta manera se tiene más fundamento en todo la investigación.

Para Valles, (2007) "Los documentos incluyen prácticamente cualquier cosa existente previa y durante la investigación" (p. 120). Ante lo cual López-Barajas, (1999) indica que "la lectura de documentos y registros resulta una tarea laboriosa y compleja”. (p. 71). Considerando que Bisquerra et al., (2004) indica que el análisis documental "requiere planificación y consiste principalmente en examinar documentos para complementar información a partir de las otras técnicas".

El análisis documental está inmerso en toda la investigación, desde el momento que se está definiendo el tema, hasta el final en la interpretación de los resultados, porque otorga bases para interpretar y conocer otros aspectos. Toma un papel importante para la investigación con la contextualización de la educación superior en Ecuador, las universidades intervenidas, toda la Ley que o leyes que se consideran a lo largo del trabajo; y en éste apartado de manera particular toma sentido con toda la información provista formalmente por las autoridades de las cinco universidades en estudio, para la revisión y organización de ésta información.

Y así está presente, inclusive en el de análisis de resultados, porque se comparan los resultados con los datos existentes en documentos para contrastar la información.

Dicho de otra manera el análisis documental está presente en toda la investigación, "las técnicas de análisis de documentos pueden ser muy elementales, como frecuencia de errores, o temas en general, hasta análisis multifactoriales. Permite descubrir tendencias, connotaciones afectivas y valores vigentes" (López-Barajas, 1999, 72). 
Las universidades estudiados a través de su representante legal, facilitaron el acceso a la información, debido a que el acceso a la información pública es reconocido en la actualidad como un derecho y como un canal del siglo XXI para la participación, la democracia y la transparencia real, que colabora en la participación efectiva y eficaz de los ciudadanos. (Cevallos, Clery, Molina, \& Santamaría, 2016, 13)

\section{Población}

La población está determinada como la totalidad de la comunidad universitaria de las cinco universidades estudiadas, que se muestran a continuación.

\begin{tabular}{|c|c|c|c|c|}
\hline Universidades en estudio & Total Docentes & Estudiantes & Total Administrativos & Total \\
\hline $\begin{array}{l}\text { Universidad Estatal del Sur } \\
\text { de Manabí }\end{array}$ & 264 & 4.404 & 267 & 4.935 \\
\hline Universidad de Guayaquil & 3.239 & 56.053 & 2.106 & 61.398 \\
\hline $\begin{array}{l}\text { Universidad Luís Vargas } \\
\text { Torres }\end{array}$ & 336 & 4.839 & 255 & 5.430 \\
\hline $\begin{array}{l}\text { Universidad Laica Eloy } \\
\text { Alfaro de Manabí }\end{array}$ & 1.320 & 16.747 & 966 & 19.033 \\
\hline $\begin{array}{l}\text { Universidad Nacional de } \\
\text { Loja }\end{array}$ & 440 & 6.688 & 488 & 7.616 \\
\hline Total & 5.599 & 88.731 & 4.082 & 98.412 \\
\hline
\end{tabular}

Fuente: Elaboración propia

Análisis de los resultados

De la información recabada en las universidades, se muestra la siguiente tabla, que pone en evidencia los presupuestos que manejan las universidades intervenidas, en su caso todas públicas, en Ecuador.

A continuación se presenta detalladamente el presupuesto asignado por parte del FOPEDEUPO a las universidades en estudio, con la descripción de: presupuesto asignado, presupuesto codificado, presupuesto ejecutado y su porcentaje de ejecución.

Presupuesto asignado: corresponde al presupuesto resultante de la fórmula de distribución de recursos, que otorga el presupuesto general del estado.

Presupuesto codificado: resulta de valores extra presupuestarios que las universidades puedan gestionar, ya sea por inversión, préstamos, donaciones

Presupuesto ejecutado: se evalúa al finalizar el ejercicio fiscal de cada año, permite identificar el nivel de gestión administrativa que tenga la universidad. 
Porcentaje de ejecución: se determina dividiendo el presupuesto codificado para el ejecutado.

Posteriormente en todos los casos se muestra gráficamente por cada año la representación de cada universidad.

La información fue recabada directamente en las universidades estudiadas. Como base sobre el uso de la información para fines académicos e investigativos, y como parte de la tesis doctoral de la autora, en el programa de Educación y Sociedad de la Universidad de Barcelona.

\section{Los criterios de análisis son:}

Incremento entre años: se analiza los presupuestos asignados a las universidades en los cuatro periodos estudiados y determinar su aumento o disminución, como variable durante el periodo de intervención.

Ejecución entre años: se valora el porcentaje de ejecución presupuestaria que ha tenido la universidad por cada año, para evidenciar la eficiencia en la gestión administrativa.

\section{Universidad Estatal del Sur de Manabí}

Tabla 3: Presupuesto Universidad Estatal Del Sur De Manabí (Unesum)

\begin{tabular}{ccccccc}
\hline Año & $\begin{array}{l}\text { Presupuesto } \\
\text { Asignado }\end{array}$ & Codificado & $\begin{array}{c}\text { Presupuesto } \\
\text { Ejecutado }\end{array}$ & \% De Ejecución \\
\hline $\mathbf{2 0 1 3}$ & $\$$ & $12.531 .038,55$ & $\$$ & $14.437 .932,57$ & $\$ 10.845 .613,87$ & 86,55 \\
$\mathbf{2 0 1 4}$ & $\$$ & $13.632 .488,87$ & $\$$ & $15.075 .429,56$ & $\$ 12.694 .573,64$ & 93,12 \\
$\mathbf{2 0 1 5}$ & $\$$ & $11.965 .260,00$ & $\$$ & $13.918 .579,04$ & $\$ 11.390 .927,52$ & 95,20 \\
$\mathbf{2 0 1 6}$ & $\$$ & $13.191 .415,00$ & $\$$ & $14.265 .501,09$ & $\$ 12.471 .163,74$ & 94,54 \\
\hline
\end{tabular}

Fuente: Elaboración propia 


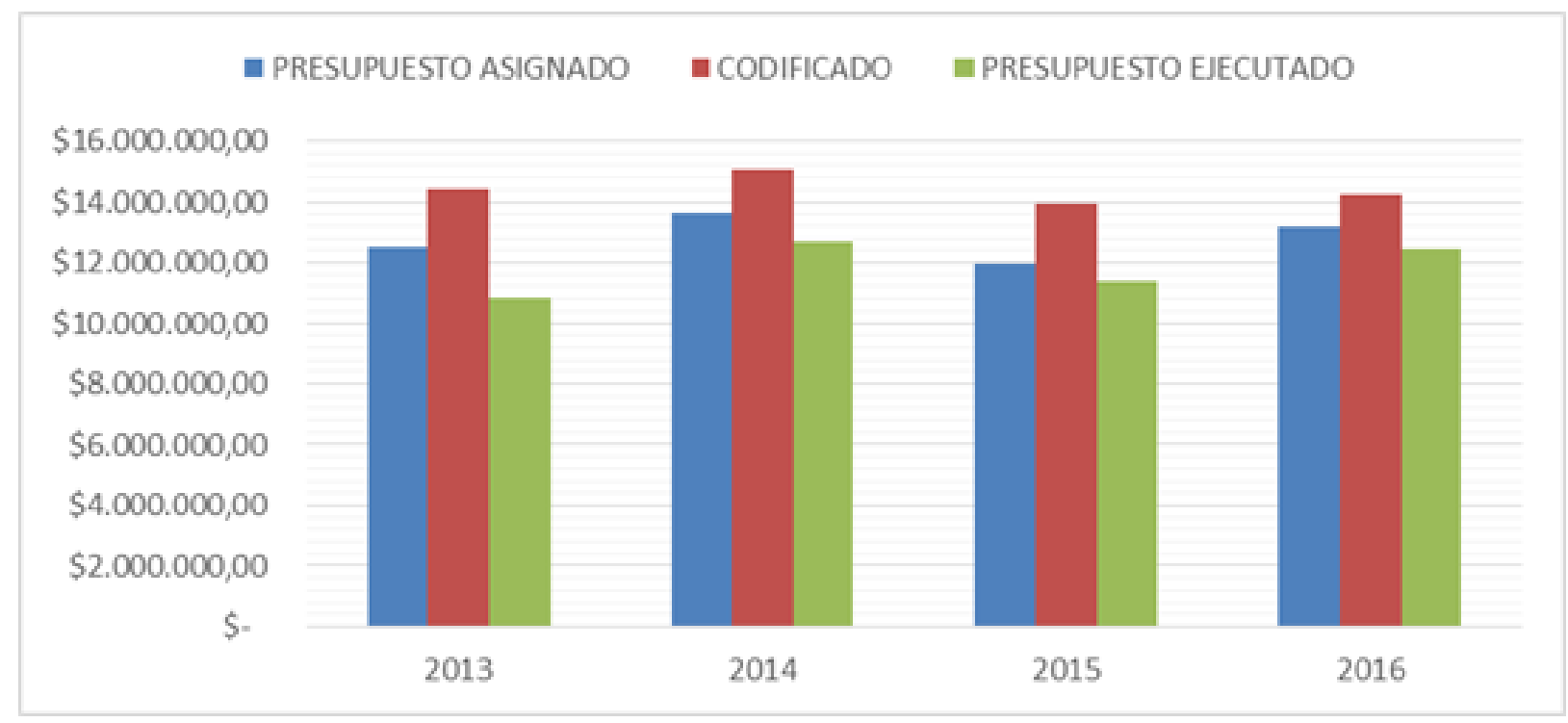

Gráfico 3. UNESUM Presupuesto acumulado por años Fuente: Elaboración propia

En el año 2013 que fue el primero de la intervención, se evidencia un presupuesto de catorce millones con una ejecución por debajo del estándar. En el periodo 2014 segundo año de intervención el presupuesto sufre una variación en aumento, así como mejora su ejecución que ya puntea índices mejores de ejecución presupuestaria. En el periodo 2015 el presupuesto vuelve a bajar (como resultado de la gestión del año 2013), sin embargo, la ejecución va en aumento y se mantiene en el 2016 cuando el presupuesto mejora, a la vez la ejecución se mantiene.

\section{Universidad de Guayaquil}

Tabla 4: Presupuesto Universidad De Guayaquil

\begin{tabular}{ccccccc}
\hline Año & Presupuesto Asignado & & Codificado & $\begin{array}{c}\text { Presupuesto } \\
\text { Ejecutado }\end{array}$ & $\begin{array}{c}\text { \% De } \\
\text { Ejecución }\end{array}$ \\
\hline $\mathbf{2 0 1 3}$ & $\$$ & $151.355 .721,34$ & $\$$ & $189.254 .603,23$ & $\$ 117.633 .666,63$ & 77,72 \\
$\mathbf{2 0 1 4}$ & $\$$ & $164.186 .214,96$ & $\$$ & $182.798 .532,39$ & $\$ 134.369 .998,32$ & 81,84 \\
$\mathbf{2 0 1 5}$ & $\$$ & $183.968 .106,00$ & $\$$ & $223.358 .080,52$ & $\$ 143.255 .964,14$ & 77,87 \\
$\mathbf{2 0 1 6}$ & $\$$ & $154.467 .331,00$ & $\$$ & $166.094 .666,67$ & $\$ 133.455 .736,33$ & 85,75 \\
\hline
\end{tabular}

Fuente: Elaboración propia 


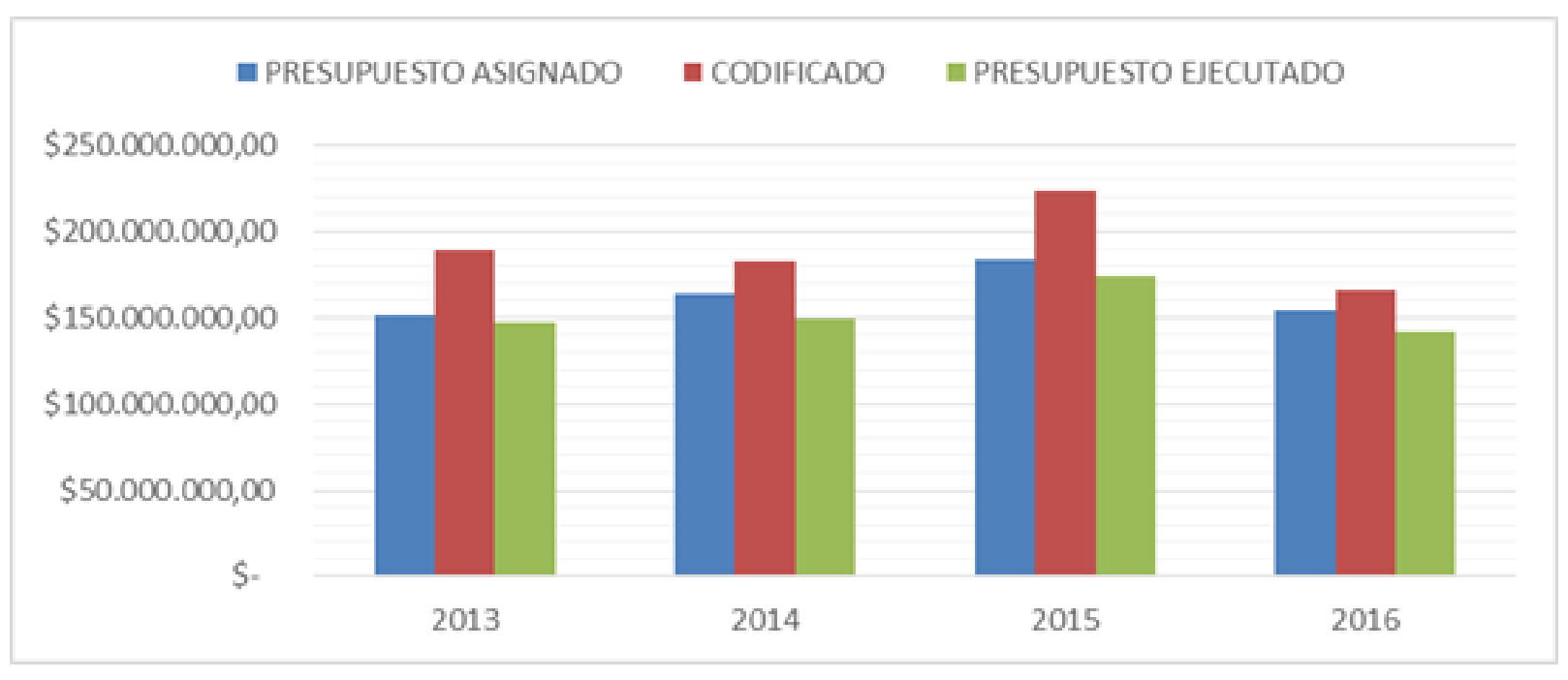

Gráfico 4: Presupuesto universidad de Guayaquil acumulado por años Fuente: Elaboración propia

En la Universidad de Guayaquil el año 2013 tuvo un presupuesto alto y con una ejecución registrada como la menor en los cuatro años analizados, y en el año 2014 su presupuesto disminuye pero su ejecución aumenta considerablemente, aún sin ser la más óptima. El 2015 registra como el año de mayores ingresos para la UG, teniendo un presupuesto por encima de los doscientos veinte y tres millones de dólares, a la vez que su ejecución disminuye en referencia al año anterior. En el 2016 nuevamente disminuye el presupuesto al nivel más bajo de todo el periodo de estudio, pero lo interesante es que la ejecución presupuestaria mejoró al nivel de ser la de mayor porcentaje durante los cuatro años estudiados.

\section{Universidad Técnica Luis Vargas Torres}

Tabla 5: Presupuesto Universidad Técnica Luis Vargas Torres

\begin{tabular}{llllllc}
\hline Año & $\begin{array}{l}\text { Presupuesto } \\
\text { Asignado }\end{array}$ & & Codificado & $\begin{array}{c}\text { Presupuesto } \\
\text { Ejecutado }\end{array}$ & $\begin{array}{c}\text { \% De } \\
\text { Ejecucion }\end{array}$ \\
\hline $\mathbf{2 0 1 3}$ & $\$$ & $21.236 .672,05$ & $\$$ & $22.624 .575,80$ & $\$ 18.728 .621,08$ & 88,19 \\
$\mathbf{2 0 1 4}$ & $\$$ & $22.650 .670,80$ & $\$$ & $26.710 .434,63$ & $\$ 18.856 .683,44$ & 83,25 \\
$\mathbf{2 0 1 5}$ & $\$$ & $20.824 .762,00$ & $\$$ & $21.051 .809,64$ & $\$ 19.527 .379,33$ & 93,77 \\
$\mathbf{2 0 1 6}$ & $\$$ & $18.625 .264,00$ & $\$$ & $18.874 .014,13$ & $\$ 18.564 .159,44$ & 99,67 \\
\hline
\end{tabular}

Fuente: Elaboración propia 


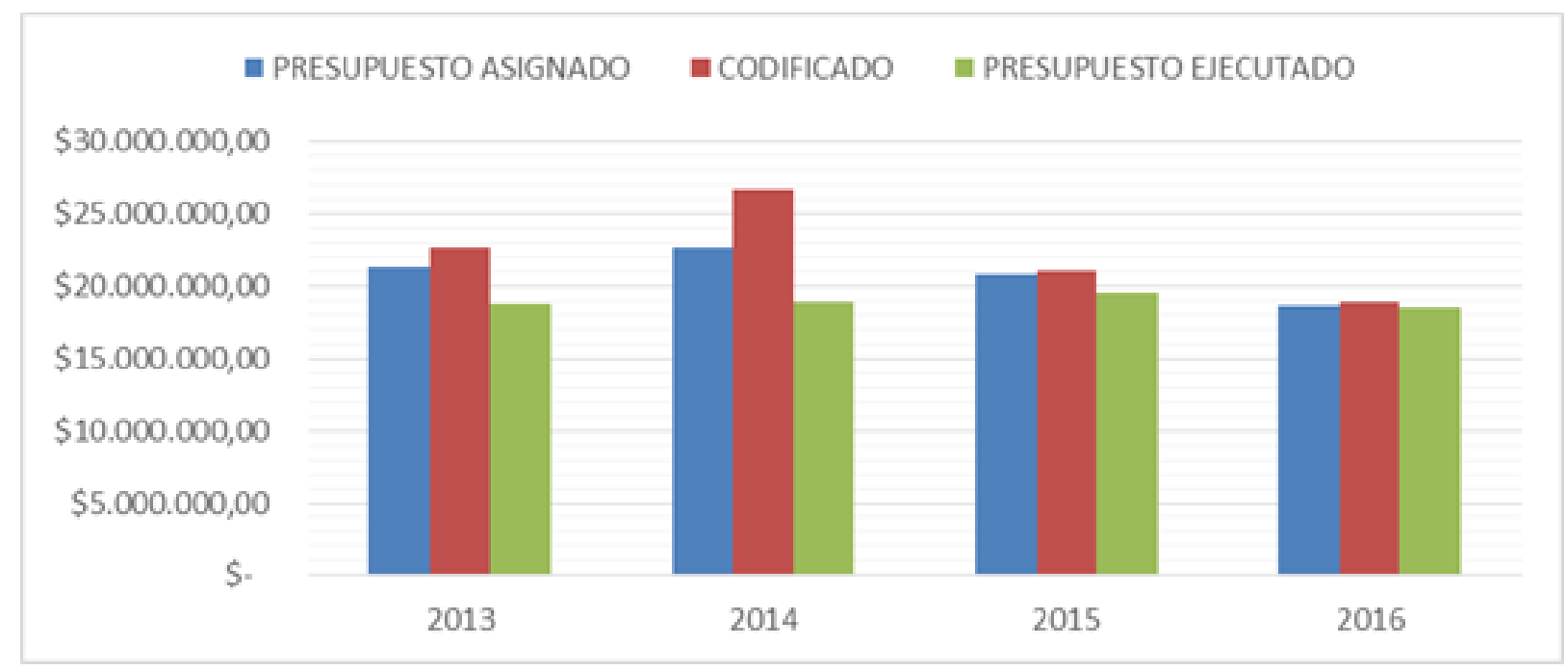

Gráfico 5. Presupuesto universidad Luis Vargas Torres acumulado por años Fuente: Elaboración propia

\section{Análisis:}

En cuanto al porcentaje de ejecución presupuestaria la UTLVT ha ido mejorando de acuerdo a los datos provistos, su presupuesto ha sido variable un 2013 con veinte y dos millones, y una ejecución por debajo de lo esperado, posteriormente en el siguiente año mejora los ingresos, pero la ejecución disminuye, significándose que la calidad de la gestión ha sido ineficiente. Y así, en el 2015 el presupuesto disminuye y su ejecución aumenta, como en el 2016 el presupuesto continúa en disminución y su ejecución mejoró al tope del nivel.

\section{Universidad Laica Eloy Alfaro de Manabí}

\section{Tabla 6: Presupuesto Universidad Laica Eloy Alfaro De Manabí}

\begin{tabular}{ccccccc}
\hline Año & Presupuesto Asignado & & Codificado & $\begin{array}{c}\text { Presupuesto } \\
\text { Ejecutado }\end{array}$ & \% De Ejecucion \\
\hline $\mathbf{2 0 1 3}$ & $\$$ & 37.062 .889 .75 & $\$$ & $48.631 .306,64$ & $\$ 34.538 .906,96$ & 93,19 \\
$\mathbf{2 0 1 4}$ & $\$$ & $43.459 .442,69$ & $\$$ & $55.086 .989,92$ & $\$ 41.647 .183,93$ & 95,83 \\
$\mathbf{2 0 1 5}$ & $\$$ & $59.726 .765,00$ & $\$$ & $61.514 .261,36$ & $\$ 55.766 .880,48$ & 93,37 \\
$\mathbf{2 0 1 6}$ & $\$$ & $58.549 .825,00$ & $\$$ & $62.219 .534,01$ & $\$ 55.165 .645,12$ & 94,22 \\
\hline
\end{tabular}

Fuente: Elaboración propia 


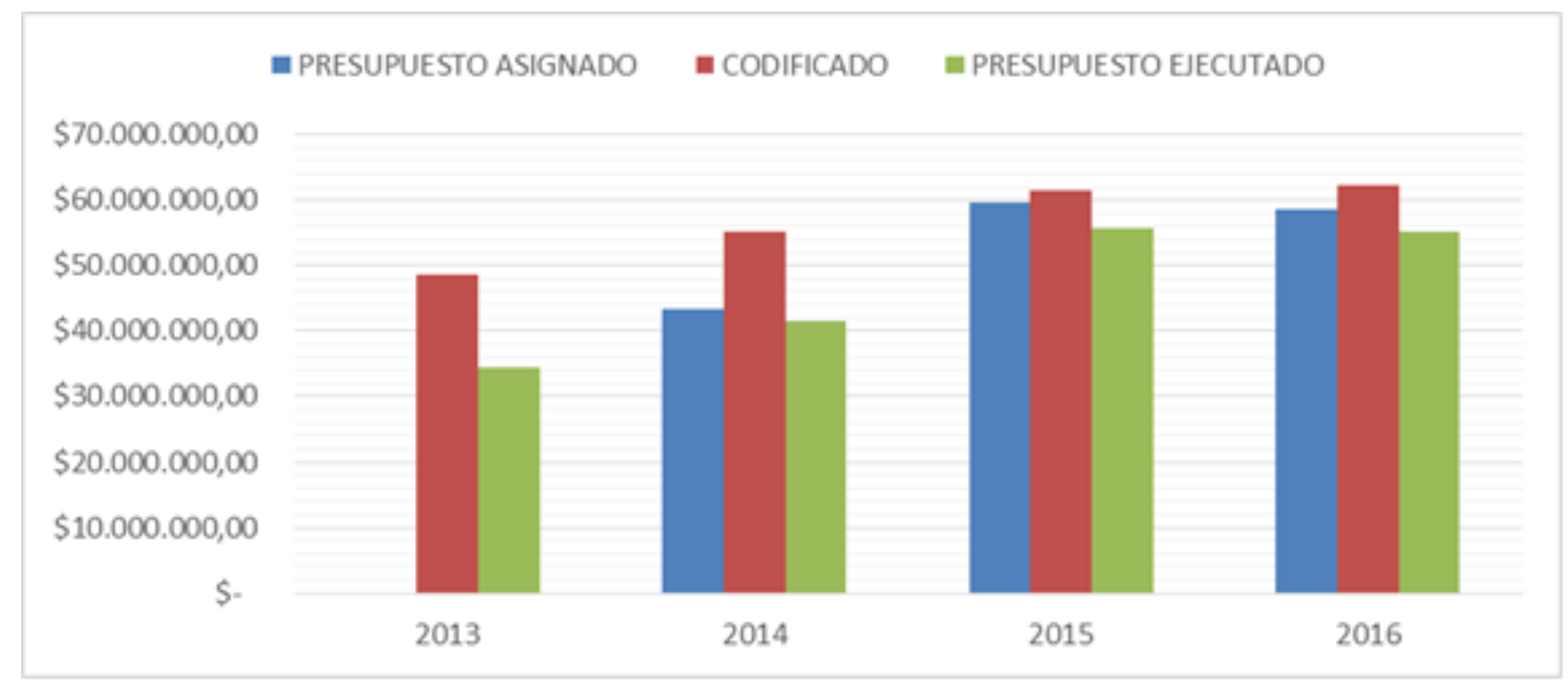

Gráfico 6. Presupuesto Uleam Acumulado por años Fuente: Elaboración propia

La ULEAM es una universidad que en términos de ejecución presupuestaria y de acuerdo a la revisión de la información, se ha mantenido por sobre el $90 \%$ situación que la fórmula de distribución de recursos valora al momento de distribuir los presupuestos, y en valores netos del presupuesto se evidencia que se ha ido incrementando cada año tanto el asignado como el codificado.

\section{Universidad Nacional de Loja}

Tabla 7: Presupuesto Universidad Nacional De Loja

\begin{tabular}{ccccccc}
\hline Año & Presupuesto Asignado & & Codificado & $\begin{array}{c}\text { Presupuesto } \\
\text { Ejecutado }\end{array}$ & $\begin{array}{c}\text { \% De } \\
\text { Ejecucion }\end{array}$ \\
\hline $\mathbf{2 0 1 3}$ & $\$$ & $42.803 .374,00$ & $\$$ & $50.736 .034,48$ & $\$ 34.499 .519,44$ & 80,60 \\
$\mathbf{2 0 1 4}$ & $\$$ & $39.454 .037,00$ & $\$$ & $55.876 .537,66$ & $\$ 33.973 .871,26$ & 86,11 \\
$\mathbf{2 0 1 5}$ & $\$$ & $40.559 .876,00$ & $\$$ & $46.623 .909,78$ & $\$ 32.476 .292,71$ & 80,07 \\
$\mathbf{2 0 1 6}$ & $\$$ & $37.979 .065,00$ & $\$$ & $39.255 .745,06$ & $\$ 28.180 .466,23$ & 74,20 \\
\hline
\end{tabular}

Fuente: Elaboración propia 


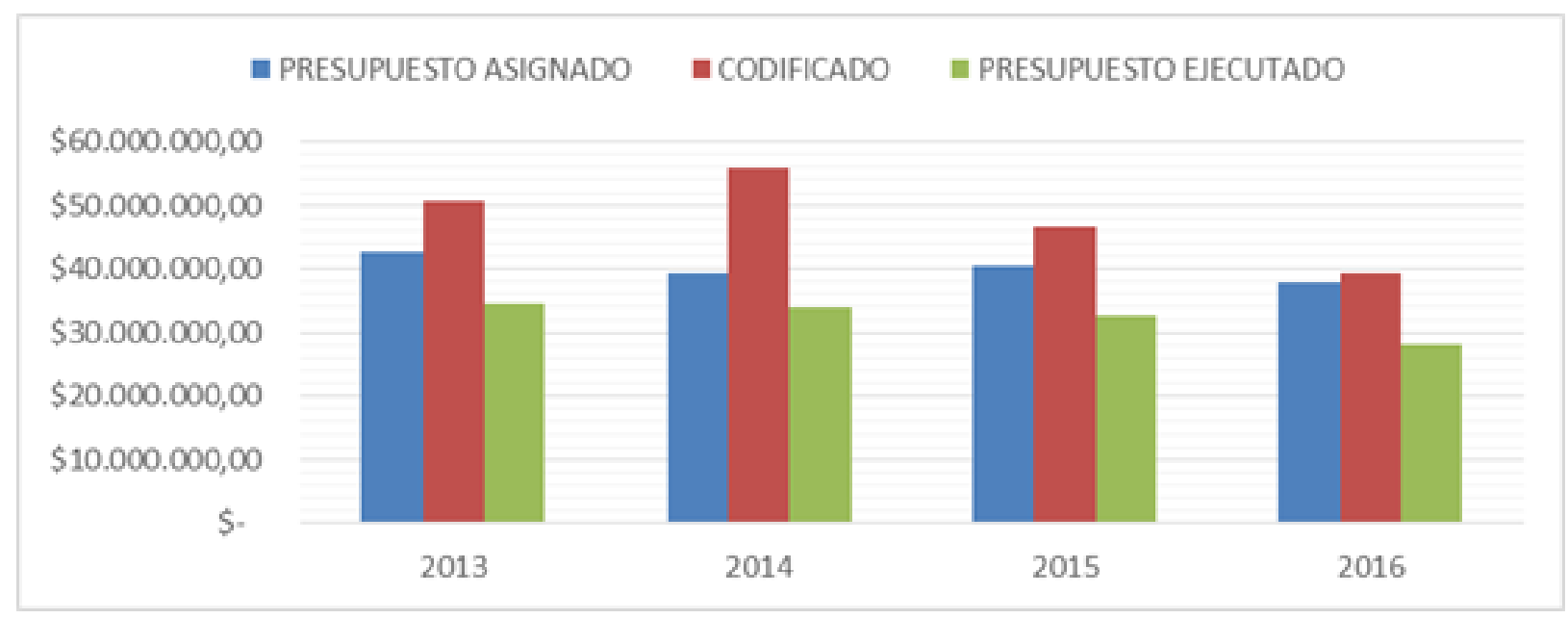

Gráfico 7. Presupuesto Universidad Nacional de Loja acumulado por años Fuente: Elaboración propia

El caso de la UNL es particular por cuanto su presupuesto ha ido teniendo variaciones con tendencia a disminuir, porque el segundo año de análisis aumenta, sin embargo en el 2015 y 2016 baja en casi diez millones de dólares, y a la vez su ejecución presupuestaria disminuye, lo que evidencia falta de gestión administrativa.

Inversión en las universidades intervenidas durante cuatro años

Tabla 8: Inversión en las universidades intervenidas durante cuatro años

\begin{tabular}{|c|c|c|c|c|c|}
\hline \multicolumn{6}{|c|}{ Presupuesto Acumulado 2013 - 2016} \\
\hline & Pre & upuesto Asignado & & Codificado & $\begin{array}{c}\text { Presupuesto } \\
\text { Ejecutado }\end{array}$ \\
\hline UNESUM & $\$$ & $51.320 .202,42$ & $\$$ & $57.697 .442,26$ & $\$ 47.402 .278,77$ \\
\hline UG & $\$$ & $653.977 .373,30$ & $\$$ & $761.505 .882,81$ & $\$ 528.715 .365,42$ \\
\hline ULVT & $\$$ & $83.337 .368,85$ & $\$$ & $89.260 .834,20$ & $\$ 75.676 .843,29$ \\
\hline ULEAM & $\$$ & $161.736 .032,69$ & $\$$ & 227.452.091,93 & $\$ 187.118 .616,49$ \\
\hline UNL & $\$$ & $160.796 .352,00$ & $\$$ & 192.492.226,98 & $\$ 129.130 .149,64$ \\
\hline TOTAL & $\$$ & 1.111.167.329,26 & $\$$ & 1.328.408.478,18 & $\$ 968.043 .253,61$ \\
\hline
\end{tabular}

Fuente: Elaboración propia

Mediante la tabla ocho se ponen en evidencia la inversión total que ha hecho el estado en las cinco universidades intervenidas en un periodo de cuatro años. (\$ 1.328.408.478,18).

\section{Conclusiones}

Se ha realizado un análisis respecto al comportamiento financiero de las universidades intervenidas por el Consejo de Educación Superior del Ecuador, desde el punto de vista teórico y desde la práctica en la gestión financiera pública que rige las políticas públicas financieras del Ecuador. 
Mediante la recopilación bibliográfica se caracterizó el sistema de educación superior en el Ecuador, poniendo énfasis en las universidades intervenidas. Por ser el objeto de estudio, sin embargo, con el análisis se tiene una visión general del funcionamiento de las universidades ecuatorianas.

El proceso de intervención es un modelo de gestión poco conocido, para ello se ha explicado sus motivaciones, sus alcances y funcionamiento mediante la normativa que lo rige y ampara en el sistema de educación superior en el país.

Las finanzas públicas es una derivación de las finanzas que estudian al sector público, para vigilar y garantizar el uso adecuado de los recursos en las entidades que se rigen en el sector público o de gobierno.

Mediante el análisis de las cédulas presupuestarias de las universidades se evidencia que el estado ha invertido $\$ 1.328^{\prime} 408.478,18$ durante los cuatro años y en las cinco universidades intervenidas, de dichos millones no se ejecutaron \$211'704.696,20 millones de dólares. Significándose que las universidades no son eficientes en su calidad de la gestión administrativa.

En la mayoría de universidades públicas la ejecución presupuestaria está ligada a la ejecución y la asignación al gasto en nómina, lo que significa que está garantizado un alto porcentaje de ejecución por ser gasto mensual en nómina.

Con el análisis de la información se pone de manifiesto que la ejecución presupuestaria aumenta en medida que el presupuesto disminuye, significándose que a menor presupuesto es más fácil para las universidades ejecutarlo.

Se evidencia además que el presupuesto codificado será siempre superior al asignado, pero el ejecutado será en todos los casos inferior a ambos. Situación preocupante que expresa que la subejecución en las universidades públicas es alto, de modo que en los cuatro años de estudio no se ejecutaron \$211'704.696,20 millones de dólares.

\section{Bibliografía}

Asamblea Nacional. Código orgánico de planificación y finanzas públicas (2010). Ecuador.

Asamblea Nacional. (2010b). Ley Orgánica De Educación Superior, 1-39.

Asensio, M. Á. (2015). Finanzas Públicas. (Osmar D. Buyatti, Ed.) (Segunda). Buenos Aires, Argentina.

Cevallos, D., Clery, A., Molina, L., \& Santamaría, G. (2016). El derecho de acceso a la información pública en la República del Ecuador. (Mar Abierto, Ed.). Manta, Ecuador.

Consejo de Educación Superior. Reglamento de aplicación de la fórmula de distribución de recursos destinados anualmente por parte del Estado a favor de las Instituciones de Educación Superior. (2017). Ecuador. Retrieved from 
http://www.ces.gob.ec/index.php?option=com_phocadownload\&view=category\&id=12\& Itemid=496\&limitstart $=20$

Córdoba Padilla, M. (2014). Finanzas públicas. (ECOE, Ed.) (Tercera). Bogotá, Colombia.

Hernández, R., Fernández, C., \& Batista, L. (2010). Metodología de la investigación. (Mc-GrawHill, Ed.). México.

Lawrence J, G., \& Chad J, Z. (2012). Principios de administración financiera (Décimosegu). (Pearson, Ed.). México.

Leopoldo, R. M. (2012). Análisis de estados financieros. (MaGrawHill, Ed.). México.

López-Barajas, E. (1999). Fundamentos de metodología científica. (Impresa, Ed.) (Cuarta rei). Madrid.

Núñez, M. (1994). Finanzas Públicas. (Macchi, Ed.). Buenos Aires, Argentina.

Rodríguez, G., Gil, J., \& García, E. (1996). Metodología de la investigación cualitativa. (Aljibe, Ed.) (Segunda). Granada.

UNESCO. (2010). UNESCO Institute for Statistics. Retrieved from http://data.uis.unesco.org/

Valles, M. (2007). Técnicas cualitativas de investigación social. Reflexión metodológica y práctica profesional. (S. S. A., Ed.). Madrid. 